\title{
An open interface in the pre-80S ribosome coordinated by ribosome assembly factors Tsr1 and Dim1 enables temporal regulation of Fap7
}

\author{
JAY RAI, ${ }^{1,6}$ MELISSA D. PARKER, ${ }^{2,6}$ HAINA HUANG, ${ }^{2}$ STEFAN CHOY, ${ }^{2}$ HOMA GHALEI, ${ }^{2,4}$ \\ MATTHEW C. JOHNSON, ${ }^{1,5}$ KATRIN KARBSTEIN, ${ }^{2,3}$ and M. ELIZABETH STROUPE ${ }^{1}$ \\ ${ }^{1}$ Department of Biological Science and the Institute of Molecular Biophysics, Florida State University, Tallahassee, Florida 32306, USA \\ ${ }^{2}$ Department of Integrative Structural and Computational Biology, The Scripps Research Institute, Jupiter, Florida 33458, USA \\ ${ }^{3}$ HHMI Faculty Scholar, Howard Hughes Medical Institute, Chevy Chase, Maryland 20815, USA
}

\begin{abstract}
During their maturation, nascent $40 \mathrm{~S}$ subunits enter a translation-like quality control cycle, where they are joined by mature $60 \mathrm{~S}$ subunits to form $80 \mathrm{~S}$-like ribosomes. While these assembly intermediates are essential for maturation and quality control, how they form, and how their structure promotes quality control, remains unknown. To address these questions, we determined the structure of an $80 \mathrm{~S}$-like ribosome assembly intermediate to an overall resolution of $3.4 \AA$. The structure, validated by biochemical data, resolves a large body of previously paradoxical data and illustrates how assembly and translation factors cooperate to promote the formation of an interface that lacks many mature subunit contacts but is stabilized by the universally conserved methyltransferase Dim1. We also show how Tsr1 enables this interface by blocking the canonical binding of elF5B to $40 \mathrm{~S}$ subunits, while maintaining its binding to $60 \mathrm{~S}$. The structure also shows how this interface leads to unfolding of the platform, which allows for temporal regulation of the ATPase Fap7, thus linking 40S maturation to quality control during ribosome assembly.
\end{abstract}

Keywords: ribosome assembly; small ribosome subunit; cryo-EM

\section{INTRODUCTION}

Ribosome assembly involves the cotranscriptional processing and folding of four ribosomal ( $r$ )RNAs that is coupled to binding of ribosomal proteins ( $r$-proteins) via a machinery of nearly 200 assembly factors (AFs). These AFs represent one way in which the cell ensures that this complex ribonucleoprotein complex assembles faithfully as the $\sim 2000$ ribosomes/minute are synthesized in a yeast cell (Warner 1999). Another mechanism by which the cell avoids dead-end or misfolded ribosomes is that ribosome assembly occurs through a series of partially assembled intermediates that serve as checkpoints, first in the nucleolus and then in the cytoplasm (Klinge and Woolford 2019). Although some intermediates were identified biochemically in the 1970s and 1980s, others have only recently

\footnotetext{
${ }^{4}$ Present address: Emory University School of Medicine, Department of Biochemistry, Atlanta, Georgia 30329, USA

${ }^{5}$ Present address: Genentech, South San Francisco, California 94080, USA

${ }^{6}$ These authors contributed equally to this work.

Corresponding authors: kkarbst@scripps.edu, mestroupe@bio. fsu.edu

Article is online at http://www.rnajournal.org/cgi/doi/10.1261/rna. 077610.120.
}

been discovered and their structures been determined, in part because of advances in cryo-EM technologies. Ribosomes are central to the cellular function across all of biology, so misfolded ribosomes severely impact the health of the cell because they can lead to errors in translation, stalling on the mRNA (Cole et al. 2009). Visualizing how those intermediates function in quality control of ribosome assembly will advance our understanding of how cells protect themselves from misfolded ribosomes.

After initial transcription and assembly in the nucleolus, largely assembled 405 precursors that retain only seven AFs (Tsr1, Dim1, Nob1, Pno1, Enp1, Ltv1, and Rio2) are exported into the cytoplasm (Baßler and Hurt 2019; Klinge and Woolford 2019). Tsr1 is a large, GTPase-like protein that shares domain homology with other GTPases involved in initiation, like elF5B or elongation like EF-Tu (McCaughan et al. 2016), but lacks the GTPase

\footnotetext{
(C) 2021 Rai et al. This article is distributed exclusively by the RNA Society for the first 12 months after the full-issue publication date (see http://rnajournal.cshlp.org/site/misc/terms.xhtml). After 12 months, it is available under a Creative Commons License (Attribution-NonCommercial 4.0 International), as described at http:// creativecommons.org/licenses/by-nc/4.0/.
} 
active site. Dim1 is a universally conserved methylase, called KsgA in bacteria, that methylates the small subunit rRNA (O'Farrell et al. 2006). Nob1 is a PIN-domain containing endonuclease that is responsible for cleavage of the $3^{\prime}$ end of the $18 \mathrm{~S}$ precursor (20S rRNA) to create the mature-length $18 \mathrm{~S}$ rRNA (Pertschy et al. 2009). Pno1 contains two tandem $\mathrm{KH}$ homology domains that bind the rRNA (Vanrobays et al. 2008) and acts as a structural partner to Nob1, regulating its endonuclease activity (Woolls et al. 2011). Enp1 and Ltv1 work together to block Rps3 from adopting its mature position to guide assembly of the 40S head (Strunk et al. 2011; Mitterer et al. 2016; Johnson et al. 2017; Collins et al. 2018). Their release from the solvent side of the pre-40S in the cytoplasm, driven by Hrr25/CK1 $\delta$ phosphorylation (Schäfer et al. 2006; Ghalei et al. 2015; Mitterer et al. 2019), commits the maturing pre-40S to a translation-like intermediate bound to mature 60S subunits (Strunk et al. 2012; Ghalei et al. 2015). Finally, Rio2 is a kinase that binds to the interface side of pre-40S, blocking the elF1A binding site to prevent premature translation but releasing before formation of the translation-like intermediate (Ferreira-Cerca et al. 2012; Strunk et al. 2012; Huang et al. 2020).

Ribosome maturation is coupled to quality control in a translation-like cycle where pre-40S ribosomes bind mature 605 subunits in an elF5B-dependent manner to produce 80S-like ribosomes (Lebaron et al. 2012; Strunk et al. 2012; Ghalei et al. 2017; Huang et al. 2020). These 80S-like intermediates preserve Tsr1 and Dim1 (Strunk et al. 2012; Ghalei et al. 2017; Shayan et al. 2020), and thus present a paradox, because structures of pre-40S ribosomes that contain the above seven AFs show that Tsr1 and Dim1 sterically block 60S binding at the interface (Strunk et al. 2011; Heuer et al. 2017; Johnson et al. 2017; Scaiola et al. 2018). Specifically, the position of Tsr1 within the stable pre-40S intermediate sterically blocks the binding of elF5B, which is required for formation of both $80 \mathrm{~S}$ and 80S-like ribosomes (Lebaron et al. 2012; Strunk et al. 2012; McCaughan et al. 2016). In addition, Tsr1 promotes a conformation of the decoding site helix, h44, which is incompatible with the binding of 60S subunits (Strunk et al. 2011; Heuer et al. 2017; Scaiola et al. 2018). However, its position is variable and contributes to heterogeneity in the pre-40S subunit (Johnson et al. 2017; Thoms et al. 2020). As it is positioned by the $3^{\prime}$ end of the rRNA, Dim1 blocks the approach of the 60S subunit, by direct overlap with $\mathrm{H} 69$ of $25 \mathrm{~S}$ rRNA. Thus, both Tsr1 and Dim1 must reposition prior to the formation of $80 \mathrm{~S}$-like ribosomes. Alternatively, or additionally, 80S-like ribosomes might have an alternate interface that is not perturbed by their position as seen in prior $40 \mathrm{~S}$ structures. Furthermore, because Dim1 sterically blocks the binding of elF1, thereby preventing premature translation initiation, its release must be regulated during ribosome assembly so translation initiation occurs only after both maturation and quality control are complete. Dim1 release occurs in 805 -like ribosomes and is catalyzed by the ribosome biogenesis ATPase Fap7 that binds Rps14 after testing their ability to faithfully translocate mRNA; bypass of this step allows release of ribosomes defective in reading frame maintenance into the translating pool (Granneman et al. 2005; Ghalei et al. 2017). Moreover, formation of $80 \mathrm{~S}$-like ribosomes is a quality control checkpoint that ensures only scanning-competent ribosomes are released into the translating pool, and bypass of these checkpoints releases ribosomes with initiation-defects into the translating pool (Huang et al. 2020). Finally, Nob1-dependent cleavage of the $3^{\prime}$ end of the rRNA occurs while this $80 \mathrm{~S}$ like is formed (Strunk et al. 2012). In summary, Tsr1 and Dim1-containing 80S-like ribosomes intermediates form in the final stages of ribosome maturation to ensure only translation-competent ribosomes enter the translation pool.

Despite their importance in quality control and maturation, the structure of 805 -like ribosomes has not been described. Thus, we do not have a structural understanding of how they are formed, despite the presence of the AFs Tsr1 and Dim1 that block subunit joining, and how their formation enables proofreading of scanning competence. Furthermore, how they enable temporal regulation of Fap7-dependent Dim1 release is also unknown. Therefore, we used single-particle cryogenic electron microscopy (cryo-EM) to visualize the structure of 805 -like ribosomes that accumulate upon Fap7 depletion (Ghalei et al. 2017). The structure is validated by biochemical data herein, as well as previous structural and biochemical data, illuminating how 80S-like ribosomes accommodate Dim1 and Tsr1, despite their position blocking subunit joining in earlier intermediates. Furthermore, structural and biochemical data also reveal how 80 S-like ribosomes enable quality control.

\section{RESULTS AND DISCUSSION}

To better understand how $40 \mathrm{~S}$ ribosome maturation is quality-controlled via structural and functional mimicry of translation events, we used cryo-EM to visualize the structure of 805 -like ribosomes. This structure was substantially different from mature $80 \mathrm{~S}$ ribosomes because the interface between the mature $60 \mathrm{~S}$ and pre-40S subunits is opened up, creating space between the subunits (Fig. 1A). This intermediate contains the interface AFs Dim1 and Tsr1, which are accommodated (together with h44) in the interface because of its opening (Fig. 1B). This significant rearrangement of the subunits answers one of the biggest questions about the structural nature of this intermediate that retains both Dim1 and Tsr1, despite their positions as steric blocks in previous pre-40S intermediates (Strunk et al. 2011; Heuer et al. 2017; Johnson et al. 2017; Scaiola et al. 2018). Tsr1 shifts modestly such that it detaches from the pre-40S head (Fig. 1C). Dim1 remains at 
A
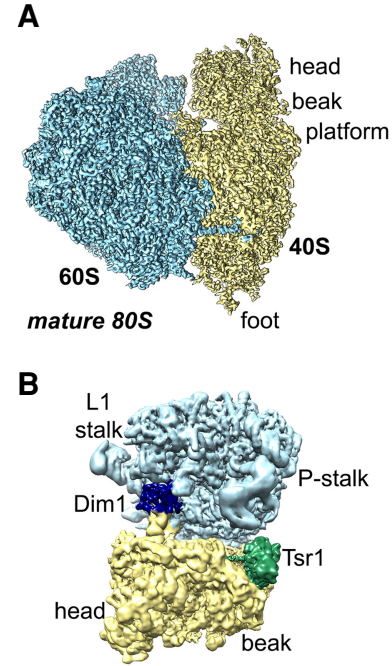

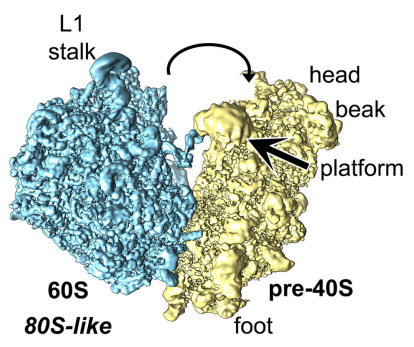

C

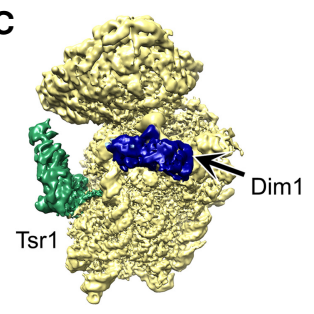

FIGURE 1. 80S-like ribosomes join via an immature interface. (A) In 80S-like preribosomes, the space between the subunits is expanded. Mature 80S/80S-like identifies each structure (bold italics). 60S/40S/ pre-40S identify each subunit (bold). Structural elements are identified in each subunit (lower case). 60S is light blue and $40 \mathrm{~S}$ and pre-40S are yellow. (B) Overall structure of 80S-like ribosomes. Dim1 is dark blue and Tsr1 is green. (C) The pre-40S interface has only the AFs Tsr1 (green) and $\operatorname{Dim} 1$ (blue) bound. The structure is viewed from the point of view of the 605 subunit.

the interface, but is removed from h45 as described below (Fig. 1C).

These ribosome assembly intermediates were accumulated by Fap7 depletion (Strunk et al. 2012; Ghalei et al. 2017) and purified via a TAP-tag on Tsr1 (Lebaron et al. 2012; Ghalei et al. 2017; Mitterer et al. 2019; Shayan et al. 2020), which is fully functional (Supplemental Fig. S1a). SDS-PAGE, mass-spectrometry, and western blot analysis demonstrate that these intermediates retain the AFs Tsr1, Dim1, and Pno1, but Nob1 is substoichiometric (Supplemental Fig. S1b,c). Additionally, 60S ribosomal proteins and ribosome associated factors like the SSA/ SSB chaperones were identified, consistent with the presence of 25S rRNA (Ghalei et al. 2017). Importantly, previous biochemical data confirmed that these intermediates are on the pathway because addition of recombinant Fap7/ATP releases Dim1 (Ghalei et al. 2017).

Initial analysis identified that two populations of particles could be visually differentiated in both raw fields of view and in 2D analysis (Supplemental Fig. S2a,b). One refined to a resolution of $3.6 \AA$ and had features like mature $80 \mathrm{~S}$ ribosomes (Supplemental Fig. S2c, d), similar to another 805 preribosome with features similar to mature ribosomes but lacking the AFs Tsr1, Dim1, and Pno1 (Scaiola et al. 2018). In this subclass, the platform, beak, decoding helix, and B3 bridge are in their mature conformation and there is no density for any tRNA (Supplemental Fig. S2e). This subclass is not described further as its significance is unclear. The other subclass had strong 60S, but weaker

pre-40S, density (Supplemental Fig. S2b). Further refinement improved the clarity of the pre-40S and 605 to overall resolutions of $3.7 \AA$ and $3.4 \AA$, respectively (Supplemental Fig. S2c,d). Although the particles showed no preferred orientation, the resolution of pre-40S remained anisotropic (Supplemental Fig. S3a-c). Nevertheless, features at the core of the particle were consistent with those overall resolution measures (Supplemental Fig. S3d). Local classification described below clarified the interface and platform to 5.5-8.5 Å-resolution (Supplemental Fig. S3e).

\section{Immature subunit interface}

One consequence of the opened 80S-like subunit interface is that fewer bridges are formed than in mature $80 \mathrm{~S}$ (Fig. 2A). For example, the B1a/b/c bridges, which involve the $40 \mathrm{~S}$ head, are not formed because the pre-40S head is far from the $60 \mathrm{~S}$ central protuberance. Similarly, pre-40S turns away from 60S on the platform side and toward it on the beak side, thus bridge B7a cannot form because that side of the head is too distant from 60S. Further, the twisted position of pre- $40 \mathrm{~S}$ relative to $60 \mathrm{~S}$ accommodates the immature position of h44, which is shifted left (Heuer et al. 2017; Scaiola et al. 2018), preventing formation of the B3 and B5 bridges at the top of h44. In contrast, eukaryote-specific bridges at the $40 \mathrm{~S}$ foot are largely maintained. In the case of a B5/B8-like bridge between h44 and Rpl23/uL14 and a B6-like bridge between h44 and Rpl24, the intersubunit connections shift due to the novel orientations of the subunits but involve analogous interactions between similar structural elements (Supplemental Fig. S4).

A striking difference between $80 \mathrm{~S}$-like and mature $80 \mathrm{~S}$ ribosomes is the interaction between $\mathrm{H} 69$ and the small subunit. In mature ribosomes, H69 binds h44 (Ben-Shem et al. 2011) to establish the B2a bridge and its deletion results in a dominant lethal phenotype (Ali et al. 2006). This bridge is the target of RRF (Pai et al. 2008), which dissociates ribosomes, as well as some antibiotics (Borovinskaya et al. 2007; Wang et al. 2012; Prokhorova et al. 2017), supporting its importance. In 80S-like ribosomes, $\mathrm{H} 69$ binds h24, which moves substantially from its mature position (Fig. 2B). Two helical densities can be assigned to H69 and h24 with high confidence, however the moderate resolution of the subunit interface prevents us from modeling atomic interactions between $\mathrm{H} 69$ and h24. The local resolution of one helix is $\sim 5.5 \AA$, lower than $60 \mathrm{~S}$ but sufficiently well-resolved to model $\mathrm{H} 69$ in its mature position (Fig. 2C; Supplemental Fig. S1e). The tip of the other helix is resolved at $\sim 8.5 \AA$; however, it connects to the well-resolved base of a repositioned h24, strongly suggesting that the RNA that binds $\mathrm{H} 69$ derives from h24 (Fig. 2C; Supplemental Fig. S1f). We validated interactions between Dim1 and h24, as well as Dim1 and 60S, in biochemical experiments described below. 
A

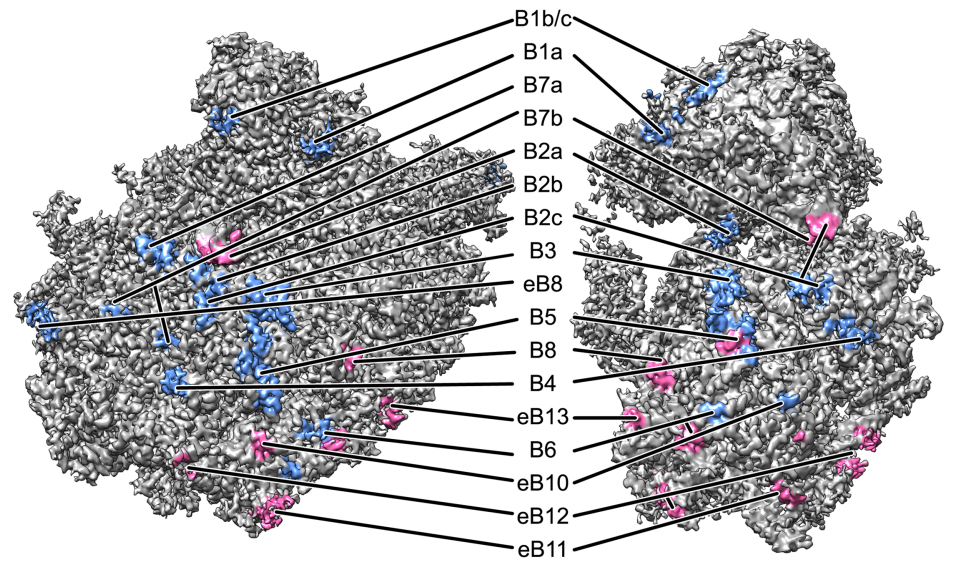

B

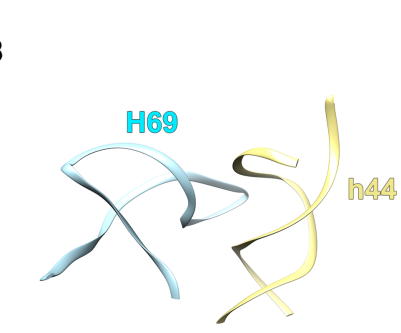

mature $80 \mathrm{~S}$

C

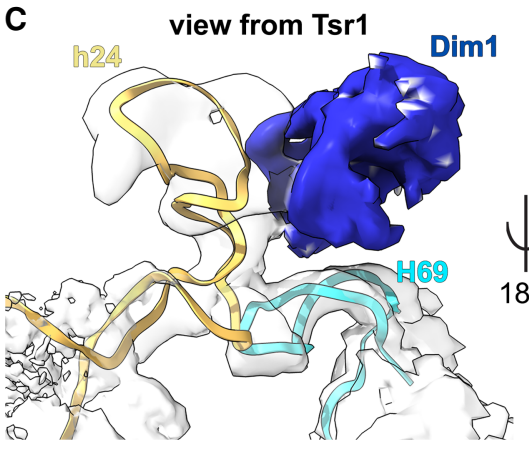

6

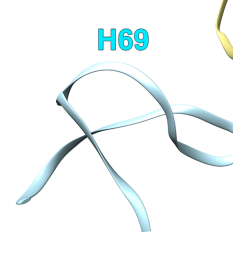

80S-like
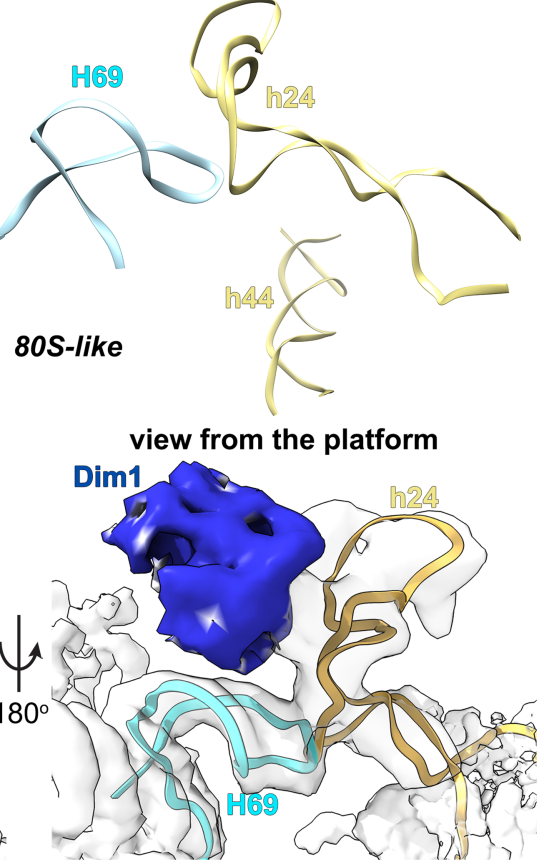

FIGURE 2. Bridges formed and not formed in 80S-like ribosomes. (A) The bridges between $60 \mathrm{~S}$ and $40 \mathrm{~S}$ head and central region of the body are largely not formed (blue patches), while those with the pre-40S foot remain intact or modestly reorganized (pink). (B) In mature ribosomes, helix 69 from the $60 \mathrm{~S}$ subunit (H69, cyan) binds helix 44 from the pre-40S subunit (h44, yellow, model from PDB ID 3J77 [Svidritskiy et al. 2014]). 80S-like ribosomes join through a novel bridge B2a/c where $\mathrm{H} 69$ (cyan) joins a repositioned h24 (yellow). (C) H69 and h24 fit into the density at the subunit interface near a bilobed density that corresponds to Dim1 (dark blue).

\section{Dim1 stabilizes h24 and binds 60S}

In pre-40S, Dim1 sterically blocks the position of the mature B2 bridge between h44 and H69, inhibiting subunit joining in canonical 80 S ribosomes (Strunk et al. 2011; Heuer et al. 2017; Johnson et al. 2017; Scaiola et al. 2018). This conflict would still exist if Dim1 remained in that position within 80 S-like ribosomes, despite their rearranged interface, because $\mathrm{H} 69$ remains the main connection between the subunits. Instead, Dim1 repositions along with h24, moving above $\mathrm{H} 69$ to alleviate the steric conflict (Fig. 3A; Supplemental Movie S1). This movement essentially releases Dim1 from pre-40S, maintaining contact only with h24, while promoting a new interaction with 60S, thus priming Dim1 for release.

We created a Dim1 variant and tested its effects on subunit joining to validate the position of Dim1, which, despite local classification, is resolved at $\sim 8.5 \AA$. The variant was designed to also probe the importance of the altered contacts between Dim1 and h24. In 80S-like ribosomes residues altered in Dim1-IKN (I250A/K253A/ N254A) face h24 (Fig. 3B). In pre-40S ribosomes, these amino acids appear to be solvent exposed (Fig. 3C; Mitterer et al. 2019). To test if the altered residues are important for formation of 805 -like ribosomes, we used an in vivo assay in which variants were expressed in a galactose-inducible/glucose-repressible Dim1/Fap7 yeast strain, supplemented with plasmids encoding wild-type or variant Dim1 and inactive Fap7. In strains competent for 80 S-like ribosome formation, accumulation of 805 -like ribosomes upon inactivation of Fap7 leads to cosedimentation of pre-18S and 25S rRNA in 80S-sized fractions (Strunk et al. 2012; Ghalei et al. 2017). Dim1-IKN expresses and shifts the equilibrium between 405 and 805 -like ribosomes away from 80S-like ribosomes (Fig. 3D,E), demonstrating a role for these residues in the formation of 805 -like ribosomes. Thus, these biochemical data validate the novel position of Dim1, and demonstrate the importance of its binding to both h24 and 60S for the formation of 80 -like ribosomes, thereby establishing a role for Dim1 in stabilizing 805 like ribosomes.

\section{Tsr1 promotes the opened interface}

In earlier 40S assembly intermediates, Tsr1 binds between the body and head, with its amino-terminal $\alpha$-helix inserted behind h44 to force it away from the body, blocking formation of canonical $80 \mathrm{~S}$ ribosomes (Supplemental Fig. S5a; Strunk et al. 2011; Heuer et al. 2017; Johnson et al. 2017; Scaiola et al. 2018). In 80S-like ribosomes, the 

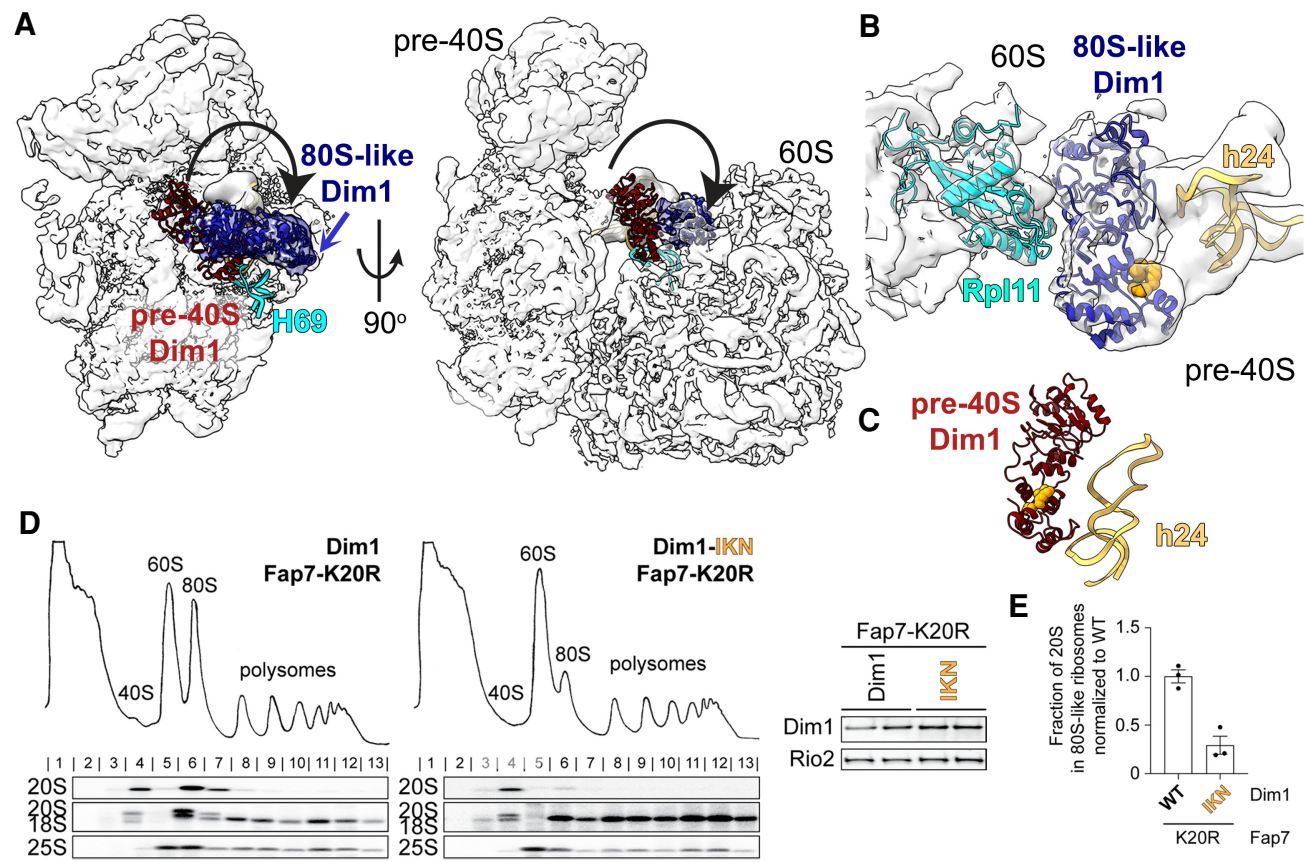

FIGURE 3. Dim1 stabilizes h24 at the 80S-like ribosome interface. (A) Local classification followed by refinement and local B-factor sharpening in Relion 3.0 revealed a bilobed density at the 60S-pre-40S interface that matches the Dim1 structure (blue). The position of Dim1 in an earlier cytoplasmic pre-40S intermediate (red, Dim 1 from PDB ID 6RBD, Mitterer et al. 2019) is also shown, demonstrating its repositioning at the interface. (B) The Dim1-IKN residues (orange) face h24. (C) In pre-40S subunits lacking 60S (PDB ID 6RBD, Mitterer et al. 2019), all altered amino acids are solvent-exposed. (D) Dim1-IKN impairs subunit joining. Whole cell extracts depleted of endogenous Dim1 and Fap7 (Gal::Dim1;Gal::Fap7) for 16-20 $\mathrm{h}$ and supplemented with plasmids encoding wild-type or mutant Dim1 and Fap7_K20R were fractionated on 10\%-50\% sucrose gradients. The sedimentation of pre-40S ribosomes containing 20S rRNA was probed by northern blot analysis using a probe directed against the unique extension of $20 \mathrm{~S}$ pre-rRNA. Mature 18S and 25S were probed with oligos directed against the mature rRNA. Note that the $18 \mathrm{~S}$ probe also picks up 20S rRNA in strains where preribosomes accumulate strongly (like the Fap7 mutants here). 80S-like ribosomes sediment in fractions 6-7 and contain $20 \mathrm{~S}$ and 25S rRNA, while pre-40S sediment in fractions 3-4 (left). Dim1 from total cell lysates was probed by western blot to demonstrate equal expression wild-type Dim1 and Dim1_IKN. Dim1 from total cell lysates was probed by western blot to demonstrate equal expression of wild-type Dim1 and Dim1_IKN from two biological replicates (right). (E) Fraction of $20 \mathrm{~S}$ in 80S-like ribosomes (fractions 6-7) compared with total $20 S$ was calculated from data in $D$ and replicates. Data were normalized to wild-type Dim1. Three biological replicates were obtained. Error bars indicate the standard error of the mean (SEM).

carboxy-terminal domain is repositioned toward the beak's tip via a rigid-body movement, pivoting around Ala63 within the amino-terminal helix, which remains behind h44 in a well-ordered region of Tsr1 (Fig. 3A; Supplemental Fig. S5b,c; Supplemental Movie S2).

To test the importance of the amino-terminal helix of Tsr1, we deleted the amino-terminal most amino acids including Ala63 (Tsr1- $\Delta$ N74) and assessed the effect on cell growth and formation of $80 \mathrm{~S}$-like ribosomes. In yeast strains where endogenous Tsr1 is under a galactose-inducible/glucose-repressible promoter, providing Tsr1- $\Delta$ N74 on a plasmid produces a strong growth phenotype (Supplemental Fig. S5d). In contrast, deleting just the amino-terminal 43 amino acids has no growth defect (Supplemental Fig. S5d). This isolates the functionally important region of Tsr1 to the helix inserted under h44.

Next, we used the in vivo subunit joining assay described above to test if the Tsr1- $\Delta$ N74 variant impairs the formation of $80 \mathrm{~S}$-like ribosomes. Sucrose-gradient fractionation of Fap7-depleted cells with wild type or Tsr1-
$\Delta N 74$ demonstrate that the ratio of $20 \mathrm{~S}$ pre-rRNA in $80 \mathrm{~S}$ like ribosomes relative to $40 \mathrm{~S}$-sized precursors is strongly reduced (Fig. 4A). This observation is supported by genetic interactions showing that Tsr1- $\Delta$ N74 behaves like another recently characterized Tsr1 mutant that blocks subunit joining, Tsr1-RK (Supplemental Fig. S5e-g; Huang et al. 2020). Tsr1-RK and Tsr1- $\Delta$ N74 are both rescued by the internal Tsr1-deletion, they are both synthetically sick with Ltv1 deletion, as well as a mutation in Rps15_YRR that affects subunit joining. Consistent with the biochemical data, these genetic interactions suggest that akin to Tsr1-RK, Tsr1- $\Delta$ N74 impairs subunit joining.

Moreover, the data also show a depletion of Tsr1- $\Delta$ N74 from $80 \mathrm{~S}$-like ribosomes, while they are bound efficiently to pre-40S ribosomes (Fig. 4B), again supporting the interpretation that this helix is required for the formation of $80 \mathrm{~S}$ like ribosomes. The increased amount of free Tsr1 in the Tsr1- $\Delta$ N74 cells is likely due to a combination of factors, including higher expression levels of Tsr1- $\Delta$ N74 (Supplemental Fig. S5h) and its inability to form 80S-like 

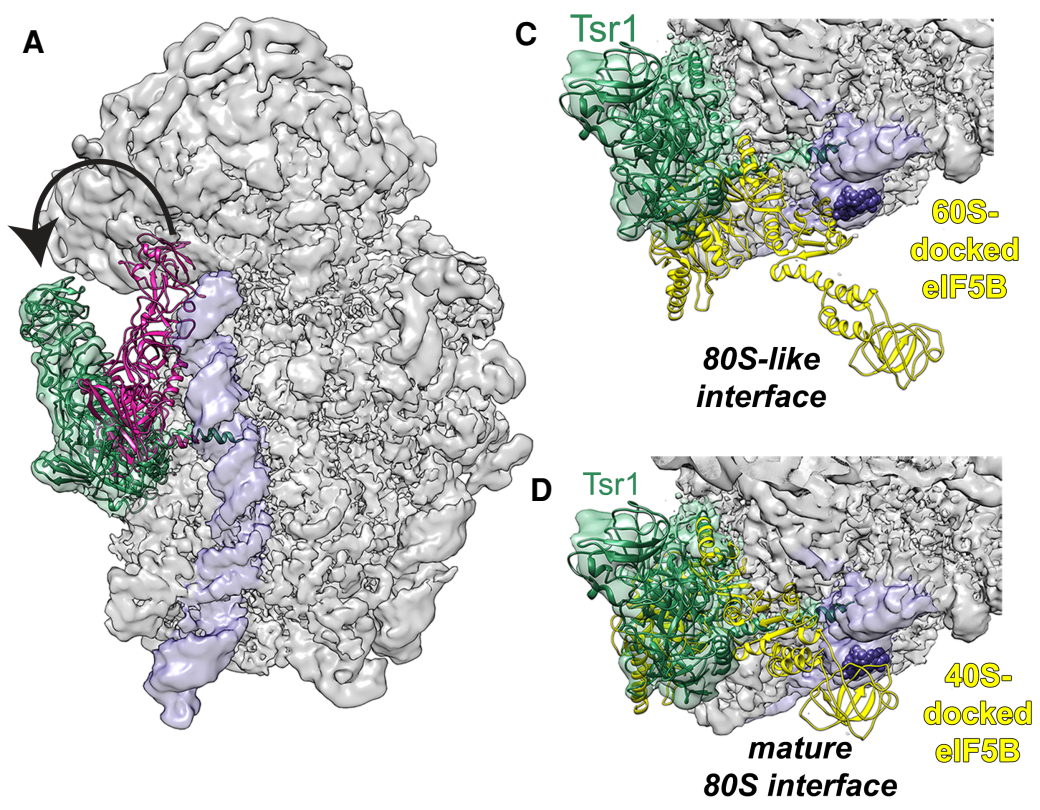

B
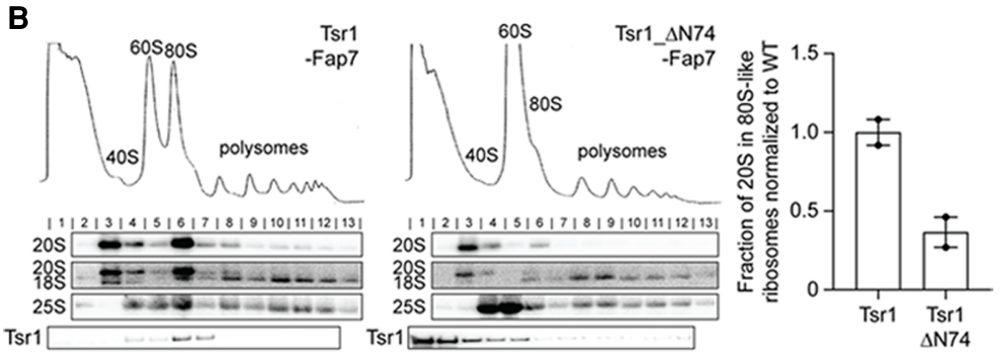

FIGURE 4. Tsr1 is repositioned to the beak. (A) The position of Tsr1 in 80S-like ribosomes (green) differs from the position in an earlier cytoplasmic pre-40S intermediate (pink, from EMD-8349 [Johnson et al. 2017]). h44 is shown in purple. (B) Whole cell extracts were fractionated as in Figure 3 (left). Quantification of the gradient northern blots (right). Fraction of $20 \mathrm{~S}$ in 805 -like ribosomes (fractions 6-7) compared with total 205 was calculated. Data are the average of two biological replicates, normalized to wild-type Tsr1, and error bars indicate the SEM. (C) The opened pre-40S and 60S interface leaves space for elF5B (yellow) across h44 (purple), blocking the early-forming B3 bridge (marked by nucleotides 1655-1657, shown as purple spheres). Model was obtained by superimposition of the $60 \mathrm{~S}$ subunits from $80 \mathrm{~S}$-like ribosomes and the elF5B-bound mature 80S ribosome (PDB ID 4V8Z [Fernández et al. 2013]). (D) If subunits were joining in the canonical mature $80 \mathrm{~S}$-structure, Tsr1 binding would block elF5B recruitment. Model was obtained by superimposition of the $40 \mathrm{~S}$ subunits from $80 \mathrm{~S}$-like preribosomes and the elF5B-bound mature 80S ribosome (PDB ID 4V8Z [Fernández et al. 2013]). The clash score, defined in Phenix (Adams et al. 2010) as the number of overlaps greater than $0.4 \AA / 1000$ atoms, increases from 170 (60S superimposition) to 780 (40S superimposition), an increase from $2 \%$ to $11 \%$ of the atoms in Tsr1.

ribosomes. Notably, weaker binding of Tsr1- $\Delta$ N74 cannot account for the observed phenotypes, because the growth of cells containing Tsr1- $\Delta$ N74 is not affected by the Tsr 1 levels (Supplemental Fig. S5h,i). Thus, our biochemical and genetic data demonstrate a role for the amino-terminal helix of Tsr1, which inserts under h44 and pivots in the transition from pre-40S to 80S-like ribosomes in the formation of $80 \mathrm{~S}$-like ribosomes, perhaps in part by facilitating this rigid body movement.

The initiation factor elF5B is required for formation of 80S-like ribosomes (Lebaron et al. 2012; Strunk et al.
2012), but its binding is incompatible with the position of Tsr1 in previously described earlier pre-40S intermediates (Strunk et al. 2011; Heuer et al. 2017; Johnson et al. 2017; Scaiola et al. 2018). To visualize if Tsr1 repositioning enables elF5B binding, we superimposed 605 from the mature $80 S \bullet$ elF5B complex onto this intermediate to place elF5B (Fig. 4C,D; Supplemental Fig. S6a,b; Fernández et al. 2013). This analysis suggests that the postulated clashes between Tsr1 and elF5B (McCaughan et al. 2016) are largely resolved in $80 S$-like ribosomes. Notably, it is the opened subunit interface that provides space for concurrent binding of elF5B and Tsr1 at the subunit interface, not Tsr1 repositioning.

In our previous data set of earlier $40 S$ assembly intermediates (Johnson et al. 2017), Tsr1 is similarly rotated in a small population of molecules (Supplemental Fig. S6c). In this lowresolution structure, Tsr1 has a disordered carboxy-terminal domain due to its mobility in isolated pre-40S and/or the low number of particles. In addition, the same position of Tsr1 is also observed in a recent structure of the SARS-CoV2 protein Nsp1 bound to pre-40S subunits (Thoms et al. 2020). Together, these observations suggest that its movement away from h44 to the beak is intrinsic to Tsr1, and not induced, for example, by elF5B.

The structure of 80S-like ribosomes clarifies multiple roles for Tsr1 in the formation of $80 \mathrm{~S}$-like ribosomes versus canonical $80 \mathrm{~S}$ ribosomes: (i) Tsr1 forces out h44, thereby sterically preventing the subunits from close approach to form the canonical subunit bridges in the head (Fig. 1A; Supplemental Fig. S5b); (ii) Tsr1 stabilizes the interface due to its interaction with both pre-40S and $60 \mathrm{~S}$ (Supplemental Fig. S6d); (iii) Tsr1 blocks access of elF5B to its typical binding site on 40S, instead enforcing a position where elF5B blocks formation of the strong and earlyforming B3 bridge (Fig. 4C,D; Lu et al. 2014). Thus, the structure suggests that by modulating the position of elF5B on the pre-40S Tsr1 steers the subunits away from the canonical B3-containing interface into 80S-like ribosomes. 


\section{Head folding in nascent pre-40S}

Previous structures show that earlier pre-40S lack Rps10/ eS10, Rps26/eS26, and Asc1 (Strunk et al. 2011; Heuer et al. 2017; Johnson et al. 2017; Scaiola et al. 2018), and that the tip of h31 remains unfolded (Heuer et al. 2017; Scaiola et al. 2018). In 80S-like intermediates, Asc1 and Rps10 locate to their mature position (Supplemental Fig. S7), indicating these proteins are recruited prior to Fap7 activity, as expected from biochemical analyses (Strunk et al. 2012). Furthermore, the tip of $h 31$ is visible (Supplemental Fig. S8). In earlier pre-40S intermediates, Tsr1 binds adjacent to the last ordered nucleotide in h31, and Rio2 binds adjacent to the h31's mature location (Strunk et al. 2011; Heuer et al. 2017; Johnson et al. 2017; Scaiola et al. 2018). Thus, we suggest that $h 31$ folding arises from dissociation of Rio 2 and detachment of Tsr1 from the head. This coincides with the beak moving to its mature position while the head straightens (Supplemental Fig. S9).

\section{Platform unfolding}

Extensive differences between earlier pre-40S subunits and 80S-like ribosomes are observed at the platform, which is opened toward $60 \mathrm{~S}$ via a rigid body motion (Fig. 1A). This movement is presumably facilitated by repositioning of h24 from the platform to the subunit interface, accompanied by disorder in the tip of h23 and partial loss of Rps1 and Rps14, as previously observed (Strunk et al. 2012), requiring local classification to improve its resolution. The other $80 \mathrm{~S}$ ribosome subclass we observe has a canonical platform (Supplemental Fig. S2e), ruling out artifacts during sample preparation as causative for the rearrangements. Further, the particles do not adopt a preferred orientation in the ice (Supplemental Fig. S3a), as expected if they were interacting with the air-water interface to unfold the platform. We therefore conclude that these changes represent folding transitions during $40 \mathrm{~S}$ subunit maturation. A recently determined structure of pre-40S, also isolated via TAP-Tsr1 but without the Fap7 deletion, describes similar unfolding of the platform (Shayan et al. 2020) but where the platform shifts toward h44, rather than opening out from the head.

To interpret the platform structure, we placed Rps $1 \bullet R p s 14 \bullet P n o 1$ from the earlier pre-40S intermediates (Scaiola et al. 2018) in the platform density with rigid body fitting, assuming the Rps14•Pno1 dimer remains unaltered (Fig. 5A). To validate this assumption, we produced mutants in the Rps14•Pno1 interface, Rps14-R107E and Pno1-QDF (Q153E/D157R/F237A, Fig. 5B). These variants were tested for growth defects in galactose-inducible/glucose-repressible Rps14 and Pno1 strains, respectively, where both demonstrated growth phenotypes (Supplemental Fig. S10a,b). Because Pno1 and Rps14 are both bound (but do not yet interact) in early 905 precursors (Bar- andun et al. 2017; Cheng et al. 2017; Sun et al. 2017), we used northern analysis to confirm that these mutations do not substantially affect early maturation events. Pno1 or Rps14 depletion reduce 20S rRNA >20-100-fold, respectively (Supplemental Fig. S10c,d; Barandun et al. 2017; Cheng et al. 2017; Sun et al. 2017). In contrast, 20 S rRNA levels are reduced approximately twofold in the Rps14-R107E and Pno1-ODF mutants, and accumulation of $23 \mathrm{~S}$ rRNA is not observed. Thus, lethal or near-lethal growth defects from these mutants do not arise from early maturation defects. Next, we used sucrose-gradient fractionation to assess if Pno1 binding to 80S-like ribosomes is affected in these mutants. 80S-like ribosomes were accumulated via depletion of Fap7 (Strunk et al. 2012), and western analysis was used to probe the sedimentation of Pno1. As expected from a weakened Pno1•Rps14 interface, the fraction of free Pno1 increases in both mutants relative to their isogenic wild-type controls (Fig. 5C,D). These data strongly suggest that the Pno1・Rps14 interface is maintained in $80 \mathrm{~S}$-like ribosomes, supporting placement of the unchanged Rps1・Rps14•Pno1 complex in the platform density. Note that Nob1 was not identified in the EM density, presumably due to its substoichiometric presence (Supplemental Fig. S1 b,c) and its mobility, which has precluded its visualization in earlier studies that did not use crosslinking (Strunk et al. 2011; Heuer et al. 2017; Johnson et al. 2017; Scaiola et al. 2018). Similarly, density corresponding to ITS1 is not visible, as also observed in previous structures (Strunk et al. 2011; Heuer et al. 2017; Johnson et al. 2017; Scaiola et al. 2018; Shayan et al. 2020).

\section{Regulation of Fap7 activity at a late stage in ribosomal assembly}

The intermediates studied here were purified from Fap7depleted cells. Consistently, addition of Fap7•ATP to these intermediates leads to Dim1 release (Ghalei et al. 2017). To confirm that the molecules can bind Fap7 stably, we developed a binding assay where we add either Fap7 or Fap7•Rps14 in the presence of either ATP or the nonhydrolyzable ATP-analog AMPPNP to the 80S-like ribosomes herein, and assay for binding by cosedimentation with ribosomes. These data show that Fap7 binds these intermediates. Addition of Rps14 stabilizes binding two- to threefold, consistent with reduced occupancy of Rps14 in the purified particles. In addition, AMPPNP stabilizes binding over ATP (Fig. 6A), consistent with ATPase-dependent release of Dim1 (and presumably Fap7) from 80S-like ribosomes (Ghalei et al. 2017). Unfortunately, we were unable to obtain a structure from these Fap7-bound ribosomes, presumably due to partial occupancy. Nonetheless, we could model Fap7 into the platform by docking the crystal structure of the Fap7•Rps14 dimer to better understand how Fap7 functions in Dim1 release. Two Fap7•Rps14 dimers were present in the asymmetric unit of the crystals 
A

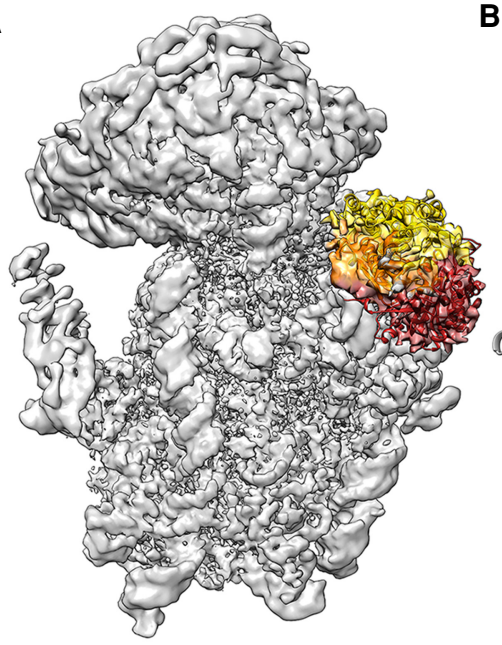

B

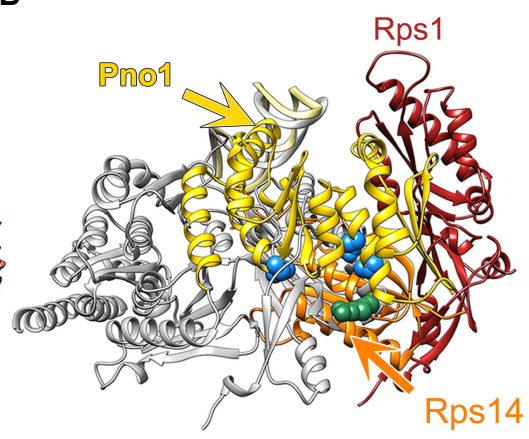

C

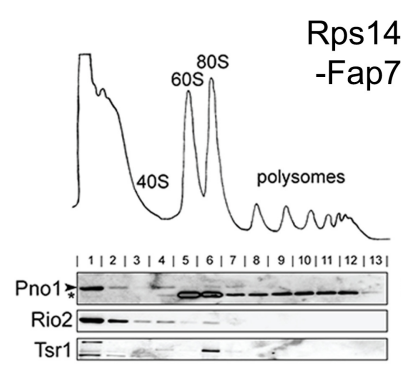

D

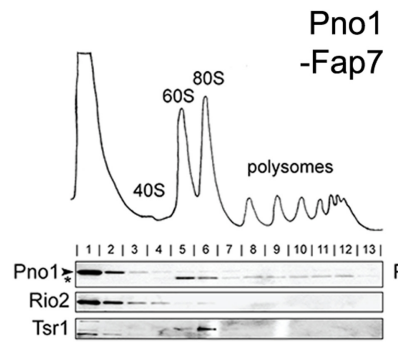

Rps14

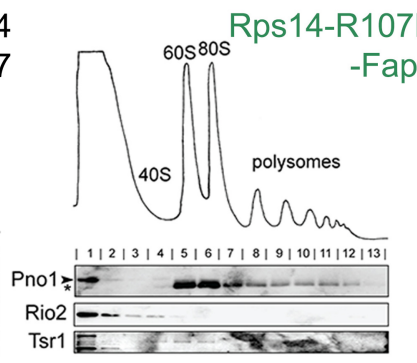

FIGURE 5. An opened pre-40S platform retains the Rps $1 \bullet$ Rps $14 \bullet$ Pno 1 trimer. (A) After local classification at the platform, alignment, and local B-factor sharpening in Relion-3.0 (Zivanov et al. 2018), the dominant class has density corresponding to Rps1 (red), Rps14 (orange), and Pno1 (yellow) albeit not at their final positions. (B) In 80S-like ribosomes Rps1 (red), Rps14 (orange), and Pno1 (yellow) are shifted outwards relative to their positions in an earlier pre-40S intermediate (PDB ID 6FAI [Scaiola et al. 2018], Rps1, Rps14, and Pno1 in gray). The residues in Pno1-QDF (Q153E; D157R; F237A, blue) and Rps14-R107 (green) are at the interface between Pno1 and Rps14. (C) The indicated whole cell extracts were fractionated as in Figure 3 and analyzed by western blot (left). Bound Pno1 was calculated as the percent of Pno1 in fractions 4-13 compared to total Pno1 (right). Data are the average of three biological replicates and error bars indicate SEM. Note that the top band is Pno1, marked with an arrow, while the bottom band, marked with an asterisk, represents cross-reactivity. $(D)$ The indicated whole cell extracts were fractionated as in Figure 3 and analyzed by western blot (left). Bound Pno1 was calculated as in C (right). Data are the average of three biological replicates and error bars indicate SEM.

(Loc'h et al. 2014), but after superimposition of Rps14, one molecule of Fap7 is positioned between Dim1 and Rps14 (Fig. 6B), consistent with biochemical data that show Fap7 bridges these proteins (Ghalei et al. 2017). Intriguingly, the second molecule of Fap7, which was originally pro-

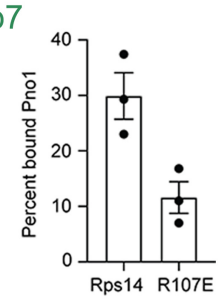

posed to be the biologically relevant dimer (Loc'h et al. 2014), clashes with the platform (Fig. 6C).

To validate the position of Fap7, we created mutations in Fap7 and Rps14 that would affect the interface that allows Fap7 to bridge Dim1 and Rps14. Rps14-K49E and Fap7-RYD (R114E/ Y116A/D118K) alter residues that contact their binding partners only in the dockable interface, whereas Rps14RVM (R41E/N42L/M46A) alters an arginine that binds Fap7 in both ( $\mathrm{Val} 42$ and Met46 bind Fap7 in the dockable interface) (Fig. 6B,C). Each mutation results in a substantial growth phenotype (Fig. 6D). Furthermore, recombinant Fap7RYD and Rps14-RVM bind Rps14 and Fap7 more weakly (Fig. 6E). Finally, as in inactive Fap7 (Ghalei et al. 2017), the growth phenotype from Fap7RYD is partially rescued by a self-releasing Dim1 mutation, Dim1-EKR (Fig. 6F). Together, these experiments support this placement of Fap7 in 80Slike ribosomes. Additionally, this biochemically validated position of Fap7 between Rps14 and Dim1 also validates interpretation of the mediumresolution features of the map: Rps14 on the platform and Dim1 at the subunit interface.

Fap7 binds Rps14 (Hellmich et al. 2013; Loc'h et al. 2014; Ghalei et al. 2017) and the biochemical data above demonstrate the importance of their interaction for $40 \mathrm{~S}$ maturation and Dim1 release (Ghalei et al. 2017). Rps14 is already bound in the earliest nucleolar 40S assembly intermediates (Barandun et al. 2017; Cheng etal. 2017; Sun et al. 2017), yet Fap7-dependent Dim1 release is one of the last cytoplasmic steps in maturation, raising the question how Fap7 recruitment to nascent ribosomes is regulated over the course of ribosome assembly. Docking of Fap7 onto earlier pre-40S intermediates answers this question because it shows that in the folded platform there is steric conflict between Fap7 and the rRNA, regardless of which Fap7•Rps14 dimer is docked onto the Rps14 position (Fig. 6G). Specifically, the Fap7 binding site on Rps14 that positions Fap7 as a bridge to Dim 1 is blocked by h23. In contrast, in 80S-like ribosomes this steric conflict is relieved by 
A
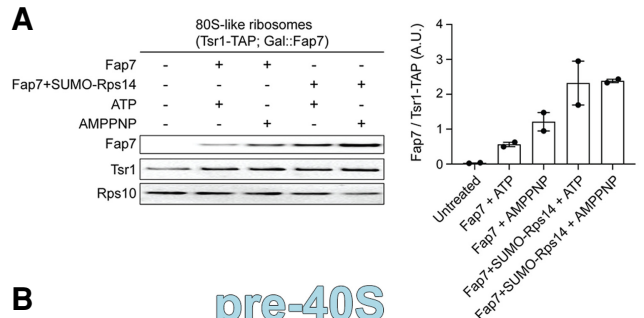

B $\quad$ 9re $=408$
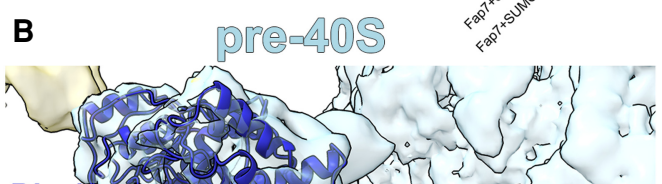

D

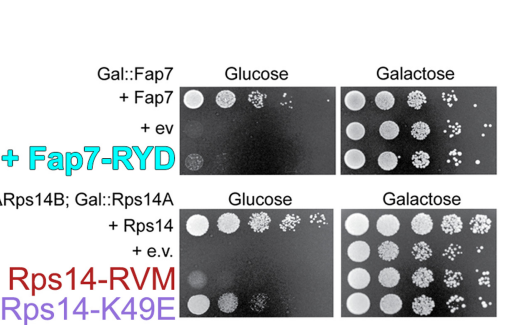

+ Rps14-K49E

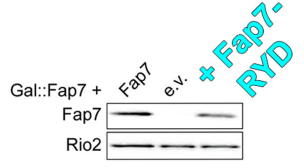

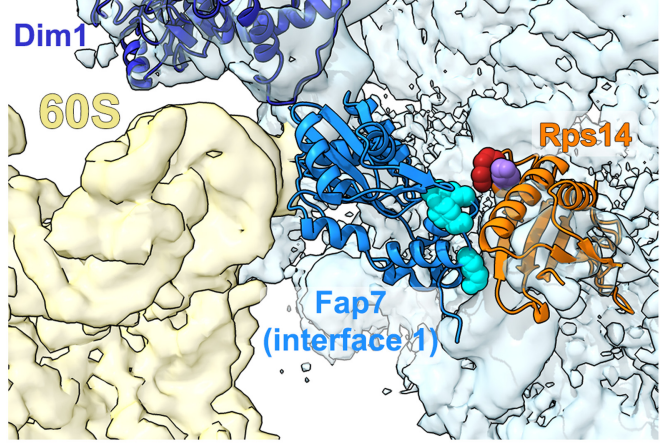

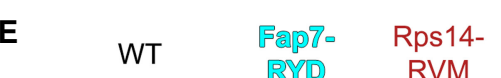
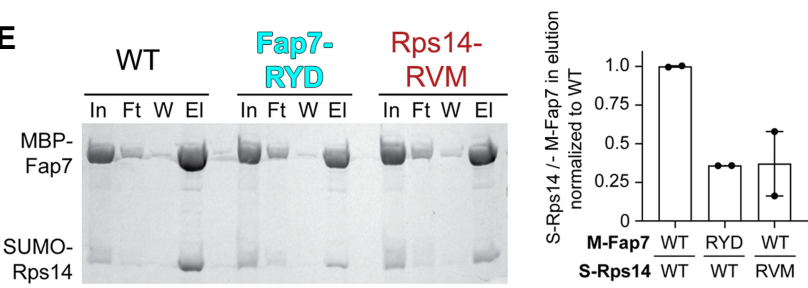

$$
R
$$
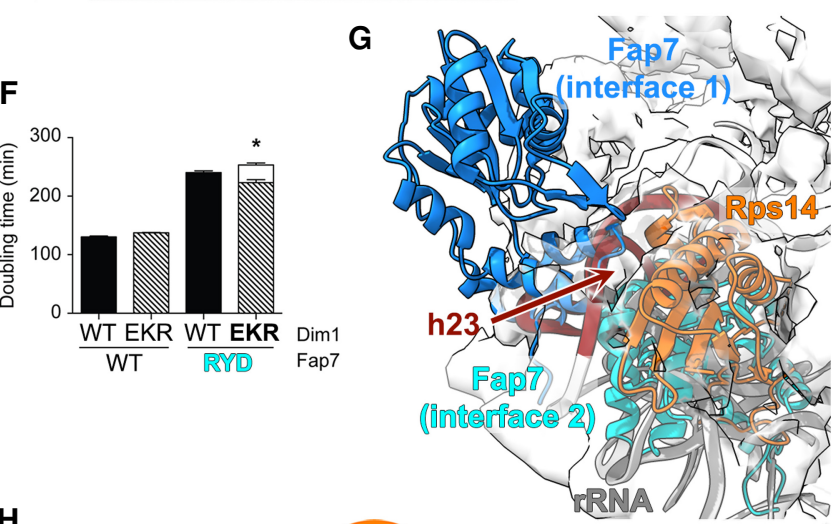

H
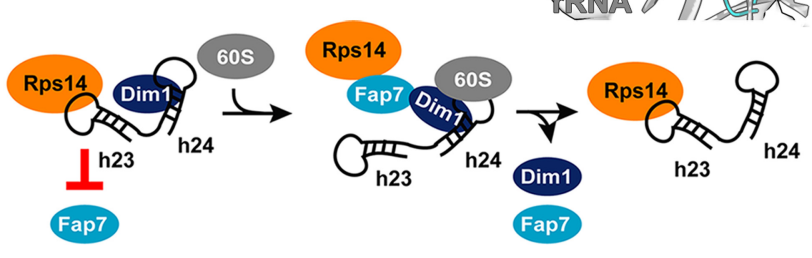

90S processosom

80S-like intermediate

mature $40 \mathrm{~S}$

FIGURE 6. Fap7 can only bind an opened platform. (A) Fap7 binds to purified 80S-like ribosomes. Fap7 binding in the presence and absence of Rps14 and ATP or AMPPNP was assessed in a pelleting assay. Ribosome pellets were probed for Fap7, Tsr1-TAP or Rps10 (left), and bound Fap7 was quantified relative to the amount of 80S-like ribosomes (Tsr1-TAP, right). Data are the average of two replicates and error bars indicate SEM. Arbitrary Units, A. U. (B) Docking Fap7•Rps14 (Fap7, chain G and Rps14, chain F) from PDB ID 4CW7 (Loc'h et al. 2014) onto Rps14 (orange) in 80S-like ribosomes places Fap7 (bright blue) in direct contact with Dim1 (dark blue), as predicted by previous biochemical data (Ghalei et al. 2017). The residues in Fap7-RYD (R114E/Y116A/D118K, cyan) and Rps14-RVM (R41E/V42L/M46A, red) are at the interface between Fap7 and Rps14 (formed between chains G and F). Rps14-K49E is in purple. In contrast, chain C from that same structure clashes with the pre-40S platform. (C) An alternative dimer of Fap7•Rps14 reveals that Fap7 (cyan) would be in steric clash with the pre-40S platform. (D) Growth (left) or Fap7 expression levels (western blot, right) of Gal::Fap7 cells containing an empty vector (e.v.), wild-type Fap7, or Fap7-RYD or growth of $\Delta$ Rps14B; Gal:: Rps14A cells containing an empty vector (e.v.), wild-type Rps14, Rps14-RVM, or Rps14-K49E plasmids were compared by 10-fold serial dilution on YPD or YPGal plates. (E) Interface mutations in Fap7 or Rps14 weaken their binding affinity for each other. Shown are Coomassie-stained SDSPAGE gels of protein binding assays on amylose beads of purified, recombinant MBP-Fap7 or MBP-Fap7-RYD and SUMO-Rps14 or SUMORps14-RVM. In, input; Ft, flow-through; W, final wash; El, elution (left). Quantification of SUMO-Rps14 (S-Rps14) compared to MBP-Fap7 (MFap7) in elution normalized to wild-type (right). Data are the average of two replicates and error bars indicate SEM. (F) Doubling time, in minutes, of cells depleted of endogenous Dim1 and Fap7 (Gal::Dim1; Gal::Fap7) and transformed with plasmids encoding Dim1 or Dim1-EKR and either Fap7 or Fap7-RYD. The white column represents the expected doubling time if there was no rescue of Fap7-RYD by Dim1-EKR (See also Ghalei et al. 2017). The height of this column was calculated by multiplying the observed fold differences for each single mutation. The data are the average of 16-17 biological replicates and the error bars represent SEM. Unpaired t-test was performed comparing expected and actual doubling times of cells expressing Fap7-RYD and Dim1-EKR. $(*)$-value $=0.030$. (G) Both Fap7 $\bullet$ Rps14 interfaces clash in pre-40S ribosomes (Rps14 [orange], h23 [red], rRNA [gray] from PDB 6FAl/EMD-4128 [Scaiola et al. 2018]). (H) The Fap7 binding site on Rps14 is blocked by h23 in nucleolar $90 \mathrm{~S}$ preribosomes and pre-40S ribosomes. 
opening of the platform, which repositions Rps14, and mobilizes h23 to expose the Fap7 binding site. Thus, unfolding of the platform in 80S-like ribosomes regulates Fap7 binding at a late stage of ribosomal assembly, when it acts to dissociate Dim1 (Fig. 6H).

80S-like ribosomes are formed in an elF5B-dependent manner during the maturation of $40 \mathrm{~S}$ ribosomes (Lebaron et al. 2012; Strunk et al. 2012). Yet, elF5B is not an essential protein in yeast, raising the question whether there are elF5B-independent pathways in $40 \mathrm{~S}$ maturation. While it is impossible to exclude such a possibility, we believe that the data herein provide evidence against this model as they demonstrate how 805 -like ribosomes are required for binding of Fap7, which is an essential protein. Thus, we believe that the nonessential nature of elF5B is simply a reflection of the ability to form 805 complexes without elF5B, which must also happen during translation in the elF5B deletion strains.

In summary, the structure of $80 \mathrm{~S}$-like ribosomes presented here, validated by genetic and biochemical data herein, as well as previous biochemical (Ghalei et al. 2017), mass spectrometry (Strunk et al. 2012), and crystallographic (Loc'h et al. 2014) data, reveal unexpected features that allow for reconciliation and explanation of many previous observations. (i) Relative to $80 \mathrm{~S}$ ribosomes, $80 \mathrm{~S}$-like ribosomes display an opened interface whose formation is enabled by Tsr1: Tsr1 supports a conformation of h44, the decoding helix, that necessitates an open interface. In addition, Tsr1 blocks the canonical binding mode of elF5B, required for formation of both 805 and $80 S$-like ribosomes; and stabilizes 80 S-like ribosomes by binding both subunits. (ii) Dim 1 is accommodated through its repositioning. In its new position it stabilizes an interaction between H69 from 605 with h24 from pre-40S, thus promoting the stability of the novel 80S-like complex interface. (iii) Because h24 is a component of the platform, the interaction between $\mathrm{H} 69$ and h24 opens the platform toward $60 \mathrm{~S}$ and mobilizes constituent RNAs and proteins. (iv) Platform remodeling allows for temporal regulation of the ATPase Fap7, which links dissociation of Dim1 to quality control (Ghalei et al. 2017). (v) As noted previously (Huang et al. 2020), the structure of the $40 \mathrm{~S}$ in $80 \mathrm{~S}$-like ribosomes resembles the structure of scanning subunits, providing a structural explanation for how formation of 805 -like ribosomes tests scanning competence to ensure the fidelity of translation initiation. Thus, this structure explains how formation of 80S-like ribosomes is required for proofreading of 405 ribosome maturation.

\section{MATERIALS AND METHODS}

\section{Yeast strains/cloning}

Yeast strains (Supplemental Table S1) were produced using PCRbased recombination (Longtine et al. 1998) and confirmed by
PCR and western blotting. Mutations in Dim1, Fap7, Tsr1, Pno1, and Rps14-expressing plasmids were introduced by site-directed mutagenesis, confirmed by sequencing.

\section{Growth assays}

Doubling times were measured in a Synergy 2 microplate reader (BioTek Instruments) as described (Collins et al. 2018; Huang et al. 2020). Statistical analyses were performed using Prism v.6.02 (GraphPad Software).

\section{Sucrose density gradient analysis}

Sucrose gradient fractionations of whole cell lysates, followed by northern and western blot analysis, were performed as described (Strunk et al. 2012). 40 S and 80 S fractions were quantified using Quantity One 1-D Analysis Software v.4.1.2 (Bio-Rad Laboratories). Pno1 was quantified using ImageJ Software v1.52 (100) (Rasband, W.S., ImageJ, U. S. National Institutes of Health, https://imagej.nih.gov/ij/, 1997-2018).

\section{Antibodies}

Antibodies against recombinant Rps10, Dim1, Nob1, Pno1, Rio2, and $\mathrm{Tsr} 1$ were raised in rabbits (Josman, LLC). Antibodies against Rpl3 or eEF2 were gifts from J. Warner or T. Goss-Kinzy, respectively.

\section{Protein binding}

MBP-Fap7(-RYD) and SUMO-Rps14(-RVM) were expressed and purified as described (Ghalei et al. 2017). Protein binding assays were performed as described (Ghalei et al. 2017). An amount of $3 \mu \mathrm{M}$ MBP-Fap7/MBP-Fap7-RYD was mixed with $4.4 \mu \mathrm{M}$ SUMO-Rps14/SUMO-Rps14-RVM in binding buffer (BB, $50 \mathrm{mM}$ Tris $\mathrm{pH} 7.5 / 150 \mathrm{mM} \mathrm{NaCl} / 5 \%$ glycerol), applied to amylose resin, washed, and bound proteins eluted in $\mathrm{BB}+50 \mathrm{mM}$ maltose.

\section{Ribosome binding assay}

80S-like ribosomes were affinity-purified from Tsr1-TAP; Gal::Fap7 cells grown in YPD medium for $16 \mathrm{~h}$ as described (Ghalei et al. 2017). An amount of $20 \mathrm{nM}$ 80S-like ribosomes were incubated with $70 \mathrm{nM}$ purified, recombinant Fap7 or Fap7+SUMO-Rps14 complex in $50 \mu \mathrm{L}$ of buffer (30 mM HEPES-KOH [pH 6.8], 100 $\mathrm{mM} \mathrm{NaCl}, 6 \mathrm{mM} \mathrm{MgCl} 2,0.5 \mathrm{mM}$ EDTA, and $1 \mathrm{mM} \mathrm{DTT).} \mathrm{ATP} \mathrm{or}$ AMPPNP was added to a final concentration of $0.5 \mathrm{mM}$. The samples were incubated on ice for $15 \mathrm{~min}$, placed on $400 \mu \mathrm{L}$ of a $20 \%$ sucrose cushion, and centrifuged for $2 \mathrm{~h}$ at 400,000 $\mathrm{g}$ in a TLA 100.1 rotor. The supernatant was removed, pellets resuspended in SDS loading dye and analyzed by western blotting.

\section{Sample purification and Cryo-EM preparation}

Fap7 was depleted by growth in YPD for $16 \mathrm{~h}$. An amount of 2.5 $\mathrm{mL}$ of lysis buffer (30 mM HEPES-KOH pH 6.8, $100 \mathrm{mM} \mathrm{NaCl}, 6$ $\mathrm{mM} \mathrm{MgCl} 2$, RNasin, PMSF, Benzamidine, EDTA-free protease 
inhibitor tablet (Santa Cruz Biotechnology), Leupeptin, Pepstatin, and Aprotinin) was added to $4 \mathrm{~g}$ of lysed frozen cell powder. The cell powder was mixed with $2 \mathrm{~mL}$ of Zircona silica beads (Millipore-Sigma) and vortexed $20 \mathrm{sec}$ for homogenization. The frozen cell lysate thawed at $4^{\circ} \mathrm{C}$ on a rocker. Thawed cell lysate was cleared via two centrifugations at $4^{\circ} \mathrm{C}$ : first, at $3000 \mathrm{~g}$ for five $\mathrm{min}$ and second, at $10,000 \mathrm{~g}$ for $10 \mathrm{~min}$. The cleared supernatant was incubated with $250 \mu \mathrm{L}$ of pre-equilibrated IgG beads (GE Healthcare) at $4^{\circ} \mathrm{C}$ for $1.5 \mathrm{~h}$ with gentle rotation. After incubation, the flow through was discarded and beads were washed three times with buffer A (30 mM HEPES-KOH pH 6.8, $100 \mathrm{mM} \mathrm{NaCl}$, $6 \mathrm{mM} \mathrm{MgCl}_{2}, 0.075 \%$ NP-40, PMSF, Benzamidine) followed by an additional wash buffer B (30 mM HEPES-KOH pH 6.8, 100 $\mathrm{mM} \mathrm{NaCl}, 6 \mathrm{mM} \mathrm{MgCl}$, PMSF, Benzamidine). Washed beads were incubated with $250 \mu \mathrm{L}$ TEV cleavage buffer $(30 \mathrm{mM}$ HEPES-KOH pH 6.8, $100 \mathrm{mM} \mathrm{NaCl}, 6 \mathrm{mM} \mathrm{MgCl}$, PMSF, Benzamidine, $1 \mathrm{mM}$ DTT and $0.5 \mathrm{mM}$ EDTA) supplemented with $2.5 \mu \mathrm{L}$ AcTEV protease (Invitrogen) at $16^{\circ} \mathrm{C}$ for $2 \mathrm{~h}$ with gentle shaking. After incubation, flow through was collected. The concentration and quality of eluate was determined spectrophotometrically using a Nanodrop 1000 (Thermo Scientific). An amount of $3 \mu \mathrm{L}$ of eluate (72 nM) was applied to a plasma-treated UltraAuFoil 1.2/1.3 grid (Quantifoil, Großlöbichau, Germany). The grids were hand blotted for $3 \mathrm{sec}$ from the backside of the grid before being plunged into liquefied ethane.

Images were acquired on a ThermoFisher/FEI Titan Krios transmission electron microscope operating at $300 \mathrm{kV}$, equipped with a DE64 camera (Direct Electron) directed by the automated hole finder in Leginon (Carragher et al. 2000). Images were recorded in "movie mode" at 1.3-2.5 $\mu$ m defocus. $25 \mathrm{e}^{-} / \AA^{2}$ dose was spread over 42 frames at a nominal magnification of $59,000 \times$, yielding $1.24 \AA$ pixel at the specimen level. 4193 micrographs were frame aligned and dose compensated with MotionCorr (Li et al. 2013). Initial CTF parameters were estimated using Gctf (Zhang 2016). A total of 146,641 particles were auto-picked in Relion-3.0, with per-particle CTF estimation/correction and beam-tilt correction (Zivanov et al. 2018). 3D classification revealed 55,949 $80 \mathrm{~S}$ particles. 90,692 particles were 80S-like ribosomes (Supplemental Fig. S2a-c; Supplemental Tables S2-S4).

Mature and 80S-like ribosomes were independently "autorefined" in Relion3.0 to 3.6 and $3.4 \AA$ A resolution, respectively. The parent $80 \mathrm{~S}$-like structure revealed an anisotropic pre-40S and was further refined with a custom mask on either subunit, yielding structures of pre-40S and $60 \mathrm{~S}$ at 3.7 and $3.4 \AA$ A-resolution, respectively (Supplemental Figs. S2d, S3a-d).

\section{Local classification/refinement}

Local classification was performed on a bin-2 stack using a spherical mask around $\mathrm{H} 69$ or the platform. For the $\mathrm{H} 69$ bridge, a dominant subclass $(43,893$ particles) was autorefined with masking on 605 to reveal the position of $\operatorname{Dim} 1$ at $\sim 7.5 \AA$ resolution (Supplemental Fig. S3e). $\mathrm{H} 69$ is resolved at $\sim 5.5 \AA$ and contours of its major and minor grooves are visible. Finally, the repositioned h24 has a resolution of $\sim 8.5 \AA$ and the twist of the helix is visible. These central elements are identifiable within the resolution limits because (i) they connect to well-resolved parts of the structure; (ii) the distinct helical contours of the RNA; and (iii) the two-domain construction of Dim1.
For the platform a dominant class $(36,914$ particles) was autorefined with a mask on pre-40S (Supplemental Fig. S3f). The platform density follows the contours of an unchanged Rps $1 \bullet$ Rps $14 \bullet$ Pno1 complex, supported by mutagenesis.

\section{Modeling}

605 and pre-40S were modeled from 805 ribosomes (PDB 3J77 [Svidritskiy et al. 2014]) using Chimera (Pettersen et al. 2004) to rigid body fit each subunit followed by manual adjustment in Coot (Emsley et al. 2010). Rpl41, h23, h45, Rps1, Rps26, and Rps14 were removed with adjustments to Rpl19, H69, and the L1 stalk. In pre-40S, h24 was moved as a rigid body to match the contours of the remodeled bridge. Additionally, the head RNA and proteins were positioned into that density. Tsr1 from PDB 6FAl (Scaiola et al. 2018) was fit as a rigid body. Yeast Dim1 was modeled in SwissModel (Waterhouse et al. 2018) and, after rigid-body fitting into the bilobed density, $\alpha$-helices and $\beta$-sheets were manually placed, followed by refinement with "Phenix.real.space.refine" (Adams et al. 2010). The model matched the density with a correlation coefficient (CC) of 0.8 at $7 \AA$ A-resolution. Rps $1 \bullet$ Rps $14 \bullet P$ no 1 was fit into the segmented platform density, matching the density with a CC of 0.6 at $7 \AA$-resolution. Each model was independently refined with "Phenix.real. space.refine" (Adams et al. 2010). Pre-40S had $89.7 \%$ residues in favored Ramachandran regions and $0.2 \%$ in outlier regions $13 \%$ Clashscore). $60 \mathrm{~S}$ had $85.9 \%$ residues in favored Ramachandran regions and $0.2 \%$ in outlier regions ( $27.9 \%$ Clashscore).

\section{SUPPLEMENTAL MATERIAL}

Supplemental material is available for this article.

\section{ACKNOWLEDGMENTS}

This work was supported by National Institutes of Health (NIH) grants R01-GM117093 and R01-GM086451, and Howard Hughes Medical Institute (HHMI) Faculty Scholar grant 55108536 to K.K. The authors acknowledge the use of instruments at the Biological Science Imaging Resource supported by Florida State University and NIH grants S10 RR025080, S10 OD018142, and U24 GM116788. H.G. was supported in part by a PGA National Women's Cancer Awareness Postdoctoral Fellowship. We wish to thank members of the Karbstein laboratory for comments on the manuscript, and Drs. Scott Stagg and Kenneth Taylor for useful discussions. Structure figures were made in ChimeraX and assembled in Photoshop (Adobe, San Jose, CA) without modification. Maps and coordinates have been uploaded to the PDB and EMDB (6WDR/21644, 6OIG/ 20077, and 20211).

Author contributions: J.R. and H.G. prepared samples; J.R. and M.E.S. carried out structure reconstruction; M.D.P., H.H., H.G., and S.C. carried out yeast analyses; M.C.J. performed cryo-EM analysis of pre-40S; K.K. and M.E.S. conceived of the experiments and wrote the paper.

Received August 12, 2020; accepted November 17, 2020. 


\section{REFERENCES}

Adams PD, Afonine PV, Bunkoczi G, Chen VB, Davis IW, Echols N Headd JJ, Hung LW, Kapral GJ, Grosse-Kunstleve RW, et al. 2010. PHENIX: a comprehensive Python-based system for macromolecular structure solution. Acta Crystallogr D Biol Crystallogr 66: 213-221. doi:10.1107/S0907444909052925

Ali IK, Lancaster L, Feinberg J, Joseph S, Noller HF. 2006. Deletion of a conserved, central ribosomal intersubunit RNA bridge. Mol Cell 23: 865-874. doi:10.1016/j.molcel.2006.08.011

Baßler J, Hurt E. 2019. Eukaryotic ribosome assembly. Annu Rev Biochem 88: 281-306. doi:10.1146/annurev-biochem-013118110817

Barandun J, Chaker-Margot M, Hunziker M, Molloy KR, Chait BT, Klinge S. 2017. The complete structure of the small-subunit processome. Nat Struct Mol Biol 24: 944-953. doi:10.1038/nsmb.3472

Ben-Shem A, Garreau de Loubresse N, Melnikov S, Jenner L, Yusupova G, Yusupov M. 2011. The structure of the eukaryotic ribosome at $3.0 \AA$ resolution. Science 334: 1524-1529. doi:10 $.1126 /$ science.1212642

Borovinskaya MA, Pai RD, Zhang W, Schuwirth BS, Holton JM, Hirokawa G, Kaji H, Kaji A, Cate JH. 2007. Structural basis for aminoglycoside inhibition of bacterial ribosome recycling. Nat Struct Mol Biol 14: 727-732. doi:10.1038/nsmb1271

Carragher B, Kisseberth N, Kriegman D, Milligan RA, Potter CS, Pulokas J, Reilein A. 2000. Leginon: an automated system for acquisition of images from vitreous ice specimens. J Struct Biol 132: 33-45. doi:10.1006/jsbi.2000.4314

Cheng J, Kellner N, Berninghausen O, Hurt E, Beckmann R. 2017. 3.2Å-resolution structure of the $90 \mathrm{~S}$ preribosome before A1 pre-rRNA cleavage. Nat Struct Mol Biol 24: 954-964. doi:10.1038/nsmb .3476

Cole SE, LaRiviere FJ, Merrikh CN, Moore MJ. 2009. A convergence of rRNA and mRNA quality control pathways revealed by mechanistic analysis of nonfunctional rRNA decay. Mol Cell 34: 440450. doi:10.1016/j.molcel.2009.04.017

Collins JC, Ghalei H, Doherty JR, Huang H, Culver RN, Karbstein K. 2018. Ribosome biogenesis factor Ltv1 chaperones the assembly of the small subunit head. J Cell Biol 217: 4141-4154. doi:10 $.1083 / j c b .201804163$

Emsley P, Lohkamp B, Scott WG, Cowtan K. 2010. Features and development of Coot. Acta Crystallogr D Biol Crystallogr 66: 486-501. doi:10.1107/S0907444910007493

Fernández IS, Bai XC, Hussain T, Kelley AC, Lorsch JR, Ramakrishnan V, Scheres SHW. 2013. Molecular architecture of a eukaryotic translational initiation complex. Science 342: 1240585. doi:10.1126/science.1240585

Ferreira-Cerca S, Sagar V, Schäfer T, Diop M, Wesseling AM, Lu H, Chai E, Hurt E, LaRonde-LeBlanc N. 2012. ATPase-dependent role of the atypical kinase Rio2 on the evolving pre-40S ribosomal subunit. Nat Struct Mol Biol 19: 1316-1323. doi:10.1038/nsmb .2403

Ghalei H, Schaub FX, Doherty JR, Noguchi Y, Roush WR, Cleveland JL, Stroupe ME, Karbstein K. 2015. Hrr25/CK18-directed release of Ltv1 from pre-40S ribosomes is necessary for ribosome assembly and cell growth. J Cell Biol 208: 745-759. doi:10.1083/jcb.201409056

Ghalei H, Trepreau J, Collins JC, Bhaskaran H, Strunk BS, Karbstein K. 2017. The ATPase Fap7 tests the ability to carry out translocationlike conformational changes and releases Dim1 during 40S ribosome maturation. Mol Cell 68: 1155. doi:10.1016/j.molcel.2017 .12 .001

Granneman S, Nandineni MR, Baserga SJ. 2005. The putative NTPase Fap7 mediates cytoplasmic 20S pre-rRNA processing through a direct interaction with Rps14. Mol Cell Biol 25: 10352-10364. doi:10.1128/MCB.25.23.10352-10364.2005
Hellmich UA, Weis BL, Lioutikov A, Wurm JP, Kaiser M, Christ NA, Hantke K, Kotter P, Entian KD, Schleiff E, et al. 2013. Essential ribosome assembly factor Fap7 regulates a hierarchy of RNA-protein interactions during small ribosomal subunit biogenesis. Proc Natl Acad Sci 110: 15253-15258. doi:10.1073/pnas.1306389110

Heuer A, Thomson E, Schmidt C, Berninghausen O, Becker T, Hurt E, Beckmann R. 2017. Cryo-EM structure of a late pre-40S ribosomal subunit from Saccharomyces cerevisiae. Elife 6: e30189. doi:10 .7554/eLife.30189

Huang H, Ghalei H, Karbstein K. 2020. Quality control of 40S ribosome head assembly ensures scanning competence. J Cell Biol 219: e202004161. doi:10.1083/jcb.202004161

Johnson MC, Ghalei H, Doxtader KA, Karbstein K, Stroupe ME. 2017. Structural heterogeneity in pre-40S ribosomes. Structure 25: 329340. doi:10.1016/j.str.2016.12.011

Klinge S, Woolford JL. 2019. Ribosome assembly coming into focus. Nat Rev Mol Cell Biol 20: 116-131. doi:10.1038/s41580-0180078-y

Lebaron S, Schneider C, van Nues RW, Swiatkowska A, Walsh D, Böttcher B, Granneman S, Watkins NJ, Tollervey D. 2012. Proofreading of pre-40S ribosome maturation by a translation initiation factor and 60S subunits. Nat Struct Mol Biol 19: 744-753. doi:10.1038/nsmb.2308

Li X, Mooney P, Zheng S, Booth CR, Braunfeld MB, Gubbens S, Agard DA, Cheng Y. 2013. Electron counting and beam-induced motion correction enable near-atomic-resolution single-particle cryo-EM. Nat Methods 10: 584-590. doi:10.1038/nmeth.2472

Loc'h J, Blaud M, Réty S, Lebaron S, Deschamps P, Bareille J, Jombart J, Robert-Paganin J, Delbos L, Chardon F, et al. 2014. RNA mimicry by the fap7 adenylate kinase in ribosome biogenesis. PLoS Biol 12: e1001860. doi:10.1371/journal.pbio.1001860

Longtine MS, McKenzie A, Demarini DJ, Shah NG, Wach A, Brachat A, Philippsen P, Pringle JR. 1998. Additional modules for versatile and economical PCR-based gene deletion and modification in Saccharomyces cerevisiae. Yeast 14: 953-961. doi:10.1002/(SICl) 1097-0061(199807)14:10<953::AID-YEA293>3.0.CO;2-U

Lu Z, Barnard D, Shaikh TR, Meng X, Mannella CA, Yassin A, Agrawal R, Wagenknecht T, Lu TM. 2014. Gas-assisted annular microsprayer for sample preparation for time-resolved cryo-electron microscopy. J Micromech Microeng 24: 115001. doi:10 .1088/0960-1317/24/11/115001

McCaughan UM, Jayachandran U, Shchepachev V, Chen ZA, Rappsilber J, Tollervey D, Cook AG. 2016. Pre-40S ribosome biogenesis factor $\mathrm{Tsr} 1$ is an inactive structural mimic of translational GTPases. Nat Commun 7: 11789. doi:10.1038/ncomms11789

Mitterer V, Murat G, Réty S, Blaud M, Delbos L, Stanborough T, Bergler H, Leulliot N, Kressler D, Pertschy B. 2016. Sequential domain assembly of ribosomal protein S3 drives $40 \mathrm{~S}$ subunit maturation. Nat Commun 7: 10336. doi:10.1038/ncomms10336

Mitterer V, Shayan R, Ferreira-Cerca S, Murat G, Enne T, Rinaldi D, Weigl S, Omanic H, Gleizes PE, Kressler D, et al. 2019. Conformational proofreading of distant $40 \mathrm{~S}$ ribosomal subunit maturation events by a long-range communication mechanism. Nat Commun 10: 2754. doi:10.1038/s41467-019-10678-z

O'Farrell HC, Pulicherla N, Desai PM, Rife JP. 2006. Recognition of a complex substrate by the KsgA/Dim 1 family of enzymes has been conserved throughout evolution. RNA 12: 725-733. doi:10.1261/ rna.2310406

Pai RD, Zhang W, Schuwirth BS, Hirokawa G, Kaji H, Kaji A, Cate JH. 2008. Structural insights into ribosome recycling factor interactions with the 70S ribosome. J Mol Biol 376: 1334-1347. doi:10 .1016/j.jmb.2007.12.048

Pertschy B, Schneider C, Gnädig M, Schäfer T, Tollervey D, Hurt E. 2009. RNA helicase Prp43 and its co-factor Pfa1 promote 20 to 
18 S rRNA processing catalyzed by the endonuclease Nob1. J Biol Chem 284: 35079-35091. doi:10.1074/jbc.M109.040774

Pettersen EF, Goddard TD, Huang CC, Couch GS, Greenblatt DM, Meng EC, Ferrin TE. 2004. UCSF Chimera-a visualization system for exploratory research and analysis. J Comput Chem 25: 16051612. doi:10.1002/jcc.20084

Prokhorova I, Altman RB, Djumagulov M, Shrestha JP, Urzhumtsev A, Ferguson A, Chang CT, Yusupov M, Blanchard SC, Yusupova G. 2017. Aminoglycoside interactions and impacts on the eukaryotic ribosome. Proc Natl Acad Sci 114: E10899-E10908. doi:10.1073/ pnas. 1715501114

Scaiola A, Peña C, Weisser M, Böhringer D, Leibundgut M, KlingaufNerurkar P, Gerhardy S, Panse VG, Ban N. 2018. Structure of a eukaryotic cytoplasmic pre-40S ribosomal subunit. EMBO J 37: e98499. doi:10.15252/embj.201798499

Schäfer T, Maco B, Petfalski E, Tollervey D, Böttcher B, Aebi U, Hurt E. 2006. Hrr25-dependent phosphorylation state regulates organization of the pre-40S subunit. Nature 441: 651-655. doi:10.1038/ nature 04840

Shayan R, Rinaldi D, Larburu N, Plassart L, Balor S, Bouyssié D, Lebaron S, Marcoux J, Gleizes PE, Plisson-Chastang C. 2020. Good vibrations: structural remodeling of maturing yeast pre-40s ribosomal particles followed by cryo-electron microscopy. Molecules 25: 1125. doi:10.3390/molecules25051125

Strunk BS, Loucks CR, Su M, Vashisth H, Cheng S, Schilling J, Brooks CL, Karbstein K, Skiniotis G. 2011. Ribosome assembly factors prevent premature translation initiation by $40 \mathrm{~S}$ assembly intermediates. Science 333: 1449-1453. doi:10.1126/science .1208245

Strunk BS, Novak MN, Young CL, Karbstein K. 2012. A translation-like cycle is a quality control checkpoint for maturing $40 \mathrm{~S}$ ribosome subunits. Cell 150: 111-121. doi:10.1016/j.cell.2012.04.044

Sun Q, Zhu X, Qi J, An W, Lan P, Tan D, Chen R, Wang B, Zheng S, Zhang $C$, et al. 2017. Molecular architecture of the 90 S small subunit pre-ribosome. Elife 6: e22086. doi:10.7554/eLife.22086
Svidritskiy E, Brilot AF, Koh CS, Grigorieff N, Korostelev AA. 2014. Structures of yeast $80 \mathrm{~S}$ ribosome-tRNA complexes in the rotated and nonrotated conformations. Structure 22: 1210-1218. doi:10 .1016/j.str.2014.06.003

Thoms $M$, Buschauer R, Ameismeier M, Koepke L, Denk T, Hirschenberger $M$, Kratzat $H$, Hayn $M$, Mackens-Kiani $T$, Cheng J, et al. 2020. Structural basis for translational shutdown and immune evasion by the Nsp1 protein of SARS-CoV-2. Science 369: 1249-1255. doi:10.1126/science.abc8665

Vanrobays E, Leplus A, Osheim YN, Beyer AL, Wacheul L, Lafontaine DL. 2008. TOR regulates the subcellular distribution of DIM2, a KH domain protein required for cotranscriptional ribosome assembly and pre-40S ribosome export. RNA 14: 20612073. doi:10.1261/rna.1176708

Wang L, Pulk A, Wasserman MR, Feldman MB, Altman RB, Cate JH, Blanchard SC. 2012. Allosteric control of the ribosome by smallmolecule antibiotics. Nat Struct Mol Biol 19: 957-963. doi:10 .1038/nsmb. 2360

Warner JR. 1999. The economics of ribosome biosynthesis in yeast. Trends Biochem Sci 24: 437-440. doi:10.1016/S0968-0004(99) 01460-7

Waterhouse A, Bertoni M, Bienert S, Studer G, Tauriello G, Gumienny R, Heer FT, de Beer TAP, Rempfer C, Bordoli L, et al. 2018. SWISS-MODEL: homology modelling of protein structures and complexes. Nucleic Acids Res 46: W296-W303. doi:10 .1093/nar/gky427

Woolls HA, Lamanna AC, Karbstein K. 2011. Roles of Dim2 in ribosome assembly. J Biol Chem 286: 2578-2586. doi:10.1074/jbc M110.191494

Zhang K. 2016. Gctf: real-time CTF determination and correction. J Struct Biol 193: 1-12. doi:10.1016/j.jsb.2015.11.003

Zivanov J, Nakane T, Forsberg BO, Kimanius D, Hagen WJ, Lindahl E, Scheres SH. 2018. New tools for automated high-resolution cryoEM structure determination in RELION-3. Elife 7: e42166. doi:10 .7554/eLife.42166 

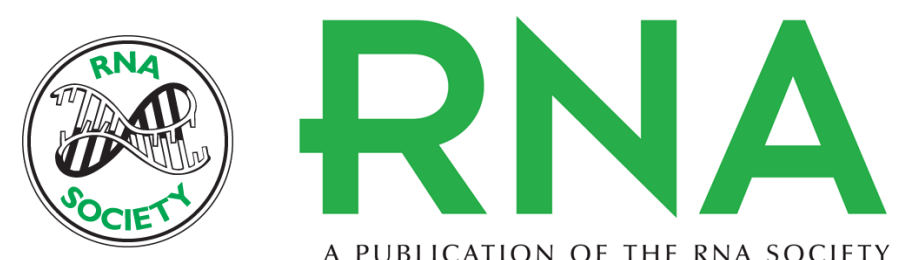

A PUBLICATION OF THE RNA SOCIETY

\section{An open interface in the pre-80S ribosome coordinated by ribosome assembly factors Tsr1 and Dim1 enables temporal regulation of Fap7}

Jay Rai, Melissa D. Parker, Haina Huang, et al.

RNA 2021 27: 221-233 originally published online November 20, 2020

Access the most recent version at doi:10.1261/rna.077610.120

\section{Supplemental http://rnajournal.cshlp.org/content/suppl/2020/11/20/rna.077610.120.DC1 Material}

References This article cites 49 articles, 14 of which can be accessed free at: http://rnajournal.cshlp.org/content/27/2/221.full.html\#ref-list-1

Creative This article is distributed exclusively by the RNA Society for the first 12 months after the Commons License full-issue publication date (see http://rnajournal.cshlp.org/site/misc/terms.xhtml). After 12 months, it is available under a Creative Commons License (Attribution-NonCommercial 4.0 International), as described at http://creativecommons.org/licenses/by-nc/4.0/.

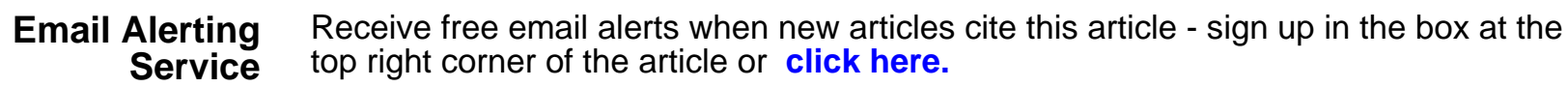

